Louisiana State University

LSU Digital Commons

Faculty Publications

Department of Physics \& Astronomy

3-1-2013

\title{
Transfer reactions and the structure of neutron-rich nuclei
}

\author{
B. P. Kay \\ University of York \\ M. Alcorta \\ Argonne National Laboratory \\ B. B. Back \\ Argonne National Laboratory \\ S. Bedoor \\ Western Michigan University \\ P. F. Bertone \\ Argonne National Laboratory
}

See next page for additional authors

Follow this and additional works at: https://digitalcommons.Isu.edu/physics_astronomy_pubs

\section{Recommended Citation}

Kay, B., Alcorta, M., Back, B., Bedoor, S., Bertone, P., Baker, S., Clark, J., Deibel, C., Di Giovine, B., Freeman, S., Hoffman, C., Lee, H., Lighthall, J., MacChiavelli, A., Marley, S., Müller, P., Pardo, R., Rehm, K., Rojas, A., Rogers, A., Rohrer, J., Santiago-Gonzalez, D., Schiffer, J., Sharp, D., Shetty, D., Thomas, J., Wiedenhöver, I., \& Wuosmaa, A. (2013). Transfer reactions and the structure of neutron-rich nuclei. Acta Physica Polonica B, 44 (3), 349-357. https://doi.org/10.5506/APhysPolB.44.349

This Article is brought to you for free and open access by the Department of Physics \& Astronomy at LSU Digital Commons. It has been accepted for inclusion in Faculty Publications by an authorized administrator of LSU Digital Commons. For more information, please contact ir@lsu.edu. 


\section{Authors}

B. P. Kay, M. Alcorta, B. B. Back, S. Bedoor, P. F. Bertone, S. I. Baker, J. A. Clark, C. M. Deibel, B. J. Di Giovine, S. J. Freeman, C. R. Hoffman, H. Y. Lee, J. C. Lighthall, A. MacChiavelli, S. T. Marley, P. Müller, R.

Pardo, K. E. Rehm, A. Rojas, A. M. Rogers, J. Rohrer, D. Santiago-Gonzalez, J. P. Schiffer, D. K. Sharp, D. V. Shetty, J. S. Thomas, I. Wiedenhöver, and A. H. Wuosmaa 


\title{
TRANSFER REACTIONS AND THE STRUCTURE OF NEUTRON-RICH NUCLEI*
}

\author{
B.P. KAY ${ }^{\mathrm{a}}$
}

for the HELIOS Collaboration

M. Alcorta ${ }^{\mathrm{b}}$, B.B. BaCK ${ }^{\mathrm{b}}$, S. Bedoor ${ }^{\mathrm{c}}$, P.F. Bertone ${ }^{\mathrm{b}}$, S.I. BAKer ${ }^{\mathrm{b}}$

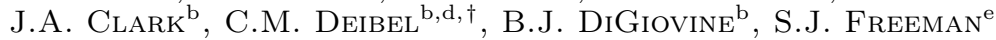

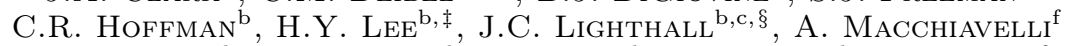
S.T. Marley ${ }^{\mathrm{b}, \mathrm{c}}$, P. MÜller ${ }^{\mathrm{b}}$, R. PARdo ${ }^{\mathrm{b}}$, K.E. Rehm ${ }^{\mathrm{b}}$, A. Rojas ${ }^{\mathrm{g}, \S}$ A.M. Rogers ${ }^{\mathrm{b}, \boldsymbol{q}}$, J. Rohrer ${ }^{\mathrm{b}}$, D. SAntiago-GonZalez ${ }^{\mathrm{g}}$ J.P. Schiffer ${ }^{\mathrm{b}}$, D.K. SharP ${ }^{\mathrm{e}}$, D.V. Shetty ${ }^{\mathrm{c}}$, J.S. Thomas ${ }^{\mathrm{e}}$ I. WiEDENHÖVER ${ }^{\mathrm{g}}$, A.H. WuOSMAA ${ }^{\mathrm{c}}$

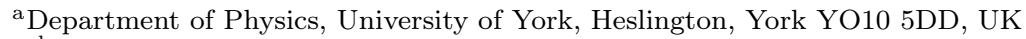

${ }^{b}$ Physics Division, Argonne National Laboratory, Argonne, IL 60439, USA

${ }^{\mathrm{c}}$ Physics Department, Western Michigan University, Kalamazoo, MI 49008, USA

d JINA, Michigan State University, East Lansing, MI 48824, USA

eschuster Laboratory, University of Manchester, Manchester M13 9PL, UK

${ }^{\mathrm{f}}$ Lawrence Berkeley National Laboratory, Berkeley, CA 94720, USA gFlorida State University, Tallahassee, FL 32306, USA

\section{(Received January 28, 2013)}

The study of transfer reactions in inverse kinematics is a major focus of existing and future radioactive-ion-beam facilities. One of the obstacles in such measurements is poor $Q$-value resolution, often several hundred $\mathrm{keV}$, which can prevent the extraction of useful information. At Argonne National Laboratory, it has recently been demonstrated that good $Q$-value resolution can be achieved by transporting the outgoing ions through a high-field solenoid, measuring their position as a function of energy. This provides several advantages over conventional Si arrays, such as large acceptance, good particle identification, and most importantly a $Q$-value resolution of better than $100 \mathrm{keV}$ in most cases, including reactions with moderately heavy beams. In this paper, the concept of the solenoidal spectrometer, called HELIOS, will be discussed along with highlights of recent results.

DOI:10.5506/APhysPolB.44.349

PACS numbers: 25.60.Je, 21.10.Pc, 29.30.Ep

* Presented at the Zakopane Conference on Nuclear Physics "Extremes of the Nuclear Landscape", Zakopane, Poland, August 27-September 2, 2012.

$\dagger$ Present address: Louisiana State University, Baton Rouge, LA 70803, USA.

$\ddagger$ Present address: Los Alamos National Laboratory, Los Alamos, NM 87544, USA.

$\S$ Present address: TRIUMF, 4004 Wesbrook Mall, Vancouver, BC V6T 2A3, Canada.

ฯ Present address: Lawrence Berkeley National Laboratory, Berkeley, CA 94720, USA. 


\section{Introduction}

Direct reactions such as single-nucleon transfer, pair transfer, and inelastic scattering, are powerful probes of nuclear structure. Key nuclearstructure information can be extracted from reaction cross sections, the angular distribution of the outgoing ions, and their energy. This includes single-particle energies, spectroscopic factors (reduced cross sections), pairing properties, and information on collective degrees of freedom. There has been a great deal learnt in exploiting these types of reactions with precision accelerators and high-resolution magnetic spectrometers over the last 50 years or so. Data from these studies has contributed significantly to our understanding of nuclear structure for stable and near-stable nuclei; however, these techniques are typically limited to stable beams and stable targets. There are a few examples where long-lived isotopes can be used as either the beam or target (for example triton beams used to be common, and ${ }^{14} \mathrm{C}$ beams or targets are possible).

As we move to the radioactive-ion-beam domain, these reactions have to be carried out in inverse kinematics, where the heavier radioactive beam impinges on a light stable target. In this regime, there are several obstacles to overcome, as discussed below, and measurements suffer from poor resolution, often several hundreds of $\mathrm{keV}$. Though challenging, the study of transfer reactions, pair transfer, and elastic scattering in inverse kinematics is well understood. As early as 1991, the first transfer reaction in inverse kinematics, $d\left({ }^{136} \mathrm{Xe}, p\right){ }^{137} \mathrm{Xe}$ at $5.868 \mathrm{MeV} / u$, was measured by Kraus et al. [1] at GSI. Outgoing protons were detected with an array of PIN-photodiodes at a fixed laboratory angle. This was, among others, followed by pioneering studies with radioactive beams such as Rehm et al. [2] measuring the $d\left({ }^{56} \mathrm{Ni}, p\right){ }^{57} \mathrm{Ni}$ reaction, and Jones et al. [3] with the $d\left({ }^{132} \mathrm{Sn}, p\right){ }^{133} \mathrm{Sn}$ both requiring major beam development studies and novel Si arrays. Many other, equally impressive, studies have been performed with radioactive beams at various facilities around the world.

Today, the most common approach for identifying and measuring the energy and angle of outgoing ions is the use of highly segmented Si detectors typically arranged in a barrel-like configuration around the target. These can be coupled with complementary, and often necessary, gamma-ray detection. Such examples are TIARA at GANIL [4], SHARC at TRIUMF [5], ORRUBA at Oak Ridge National Laboratory [6], TREX at ISOLDE [7], and MUST(2) at GANIL [8]([9]). Though not always, measurements with these composite devices rely on the use of thick targets $\left(>500 \mu \mathrm{g} / \mathrm{cm}^{2}\right.$, often $\sim 1 \mathrm{mg} / \mathrm{cm}^{2}$ ) to compensate for the efficiency loss when using large Ge detector arrays. With targets this thick, the charged-particle resolution is very poor and necessitates coincident gamma-ray gating to isolate a level. There are notable cases where this technique does not work such as decay 
from isomeric states, $0^{+} \rightarrow 0^{+}$transitions, transfer to the ground state, etc. There are also additional uncertainties in the extracted cross sections due to feeding that may not be observed, and absolute calibration of the Ge efficiency, though the latter is typically small compared to the others. A way of sidestepping some of these issues has been realised in the development of a solenoidal spectrometer called HELIOS [10] at Argonne National Laboratory (ANL). In this paper, the challenges of transfer in inverse kinematics is discussed along with a description of the HELIOS spectrometer and highlights of recent results.

\section{Transfer reactions in inverse kinematics}

The key aims in studying transfer reactions in inverse kinematics are to achieve a sufficiently good $Q$-value resolution to extract the necessary information and carry out the experiment in a reasonable timeframe. The latter demands a large acceptance device with excellent efficiency as radioactive beams are often many orders of magnitude less intense than stable beams - the lower limit being around $10^{4}$ ions per second. This explains the $\mathrm{Si}$ barrel arrangements often covering a significant fraction of the total solid angle.

The $Q$-value resolution is largely a consequence of the kinematics, if target thickness effects and intrinsic resolution are assumed fixed. First, the outgoing ions are typically low in energy due to the strong kinematic shift. This can be seen in Fig. 1 for the $(d, p)$ reaction on ${ }^{136} \mathrm{Xe}$ at forward centreof-mass (c.m.) angles. This can present problems for particle identification through standard $\Delta E-E$ techniques. The strong kinematic dependence of energy on angle requires good angular resolution, typically $<0.5^{\circ}$ in the lab. frame, which necessitates high-granularity Si arrays. Kinematic compression is another major contribution to the $Q$-value resolution. This is worst at forward c.m. angles, where the cross sections peak and most measurements are made. This effect is also illustrated in Fig. 1 and shows that the measured excitation-energy spectrum is compressed by a factor of $\sim 3$ in the laboratory frame. This is a measure of the degree to which the resolving power is lost, and effectively results in the $Q$-value resolution a factor $\sim 3$ worse. For most reactions, the kinematic compression varies between a factor of 2-4. The combination of these effects with those of the target thickness, beam properties, and intrinsic Si-detector resolution, typically results in a $Q$-value resolution of $\sim 300-400 \mathrm{keV}$ e.g. Ref. [3], though in some cases, much better has been achieved e.g. Ref. [11]. A more detailed description of these effects is given in Ref. [12] and a general description of reaction kinematics in Ref. [13]. 

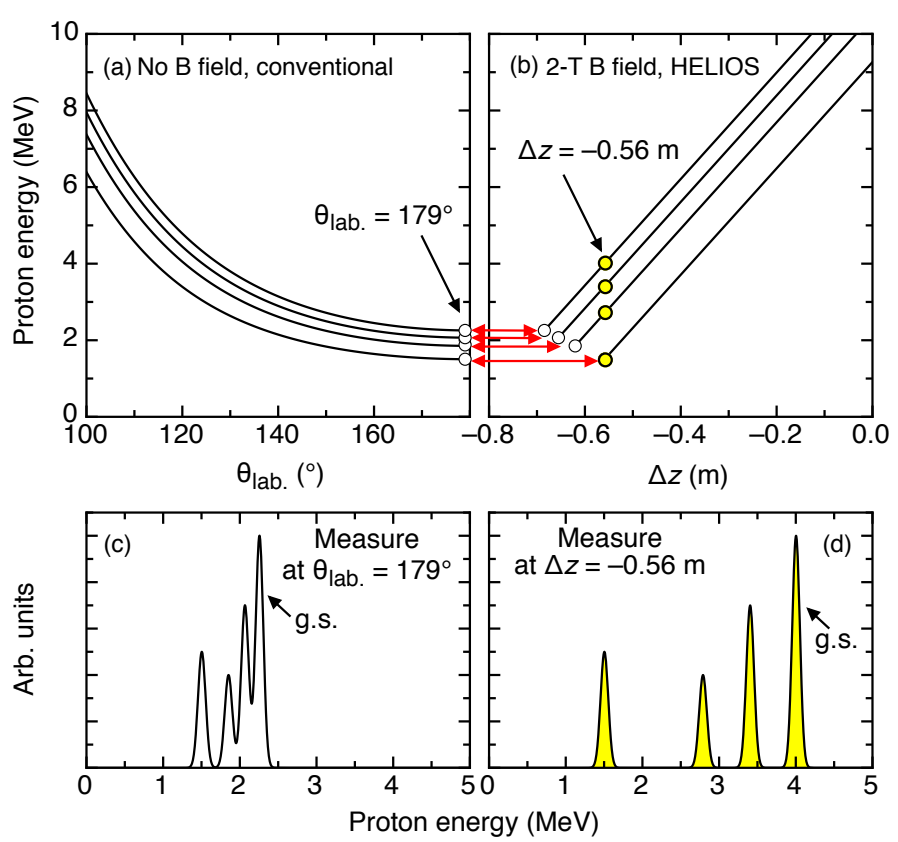

Fig. 1. Kinematic lines for low-lying states in ${ }^{137} \mathrm{Xe}$ following the $(d, p)$ reaction on ${ }^{136} \mathrm{Xe}$ in inverse kinematics at $10 \mathrm{MeV} / u$. A significant kinematic compression occurs at forward c.m. angles (a) and the resulting spectrum using conventional approaches will be compressed by being a factor of $\sim 3$ (c). In HELIOS, no kinematic compression occurs (b) and the resultant $Q$-value spectrum is not compressed for a projection at a given $\Delta z$, the distance between the target and the point at which the proton hits the detector $(\mathrm{d})$.

The HELIOS spectrometer [10, 14] exploits a different approach which overcomes some of the complications discussed above. The outgoing ions are transported in the homogeneous magnetic field of a superconducting solenoid. After leaving the target, the outgoing ions execute helical trajectories, returning to the magnetic axis (coincident with the beam axis), after one cyclotron period. Surrounding the axis is an array of position-sensitive Si detectors, which record the ions position, energy, and time with respect to the radio-frequency structure of the beam. These three properties are sufficient to provide all the information needed to fully describe the reaction. A schematic of the device is shown in Fig. 2.

In this approach, there is no kinematic compression. The excitation energy in the laboratory frame is related to the c.m. frame by only an additive constant [10]. The kinematic shift in energy versus position $(\Delta z)$ is linear and small. For a typical $(d, p)$ measurement at $2 \mathrm{~T}$, this slope is $<15 \mathrm{keV} / \mathrm{mm}$ - a position resolution of $\sim 1 \mathrm{~mm}$, the resolution of the present Si array, is 


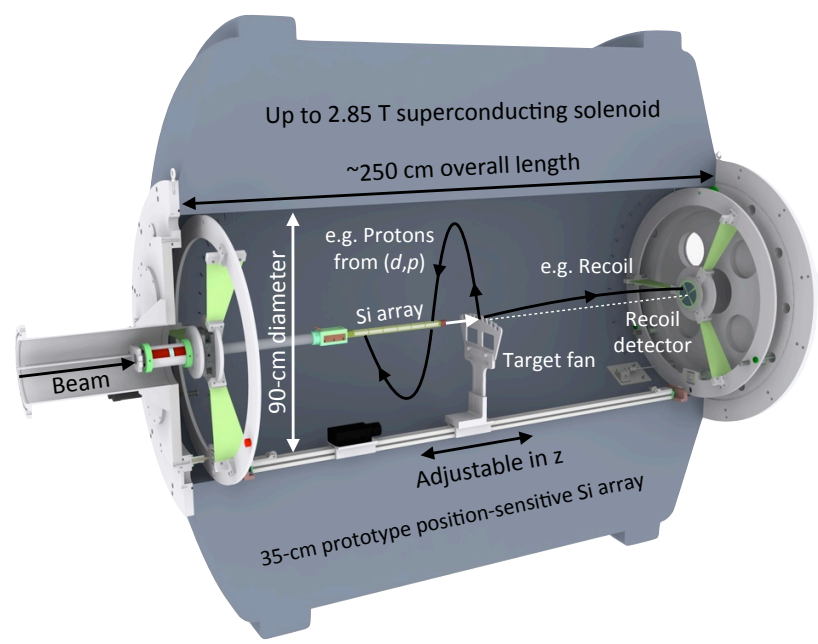

Fig. 2. Schematic of the HELIOS spectrometer modified from that in Ref. [14]. The beam enters from the left. An example proton trajectory is shown and a recoiling ion.

sufficient for the $Q$-value resolution not be dominated by position resolution. Another advantage is that the cyclotron period of outgoing ions, which is independent of their energy, is used as particle identification through the separation in time of ions with different $A / q$, and as such ions with energies as low as a few hundred $\mathrm{keV}$ can be readily identified.

Though both the conventional approach and this solenoidal spectrometer approach are limited by the target thickness and the intrinsic Si-detector resolution, the conventional approach would still suffer a poorer $Q$-value resolution as a consequence of kinematic compression. This is essentially a measure of the resolving power. The HELIOS spectrometer was commissioned in 2008 with a stable beam through the $d\left({ }^{28} \mathrm{Si}, p\right)$ reaction [14]. A $Q$-value resolution of $\sim 100 \mathrm{keV}$ was achieved.

\section{Highlights from the HELIOS program}

\subsection{In-flight beams and the neutron-rich sd shell}

Radioactive beams produced via the in-flight technique [15] at ATLAS has allowed for access to neutron-rich $s d$-shell nuclei combined with the good $Q$-value resolution of the HELIOS spectrometer. The first in-flight beam tuned to HELIOS was ${ }^{12} \mathrm{~B}$ to study excited states in ${ }^{13} \mathrm{~B}$ via ${ }^{12} \mathrm{~B}(d, p)[16]$. The focus was to determine spin-parity of the 3.48 and $3.68 \mathrm{MeV}$ states which were assumed to the be low-lying $s$ and $d$ states, but their order and 
relative strengths were not known. A previous experiment, also performed at ANL with an in-flight produced beam, but with a more conventional approach of using annular Si detectors, had failed to resolve these two states due to a $Q$-value resolution of $\sim 250 \mathrm{keV}$ [17]. A $150 \mu \mathrm{g} / \mathrm{cm}^{2} \mathrm{CD}_{2}$ target was used. The HELIOS measurement was carried out at $6.24 \mathrm{Mev} / u$ and $1.05 \mathrm{~T}$ [16]. Here a thin, $73 \mu \mathrm{g} / \mathrm{cm}^{2}, \mathrm{CD}_{2}$ target was used and a resolution of $\sim 100 \mathrm{keV}$ FWHM achieved. The two states were resolved and showed that the $s$ and $d$ orbitals are inverted at ${ }^{13} \mathrm{~B}$, which is not readily described by shell-model calculations [16]. Such a measurement would be challenging using conventional approaches. This has recently been followed up by a measurement of the $d\left({ }^{13} \mathrm{~B}, p\right)$ reaction, with the beam produced in-flight via the ${ }^{9} \mathrm{Be}\left({ }^{14} \mathrm{C},{ }^{13} \mathrm{~B}\right){ }^{10} \mathrm{~B}$ reaction, yielding $15.7-\mathrm{MeV} / u{ }^{13} \mathrm{~B}$ ions. Results of the measurement are forthcoming. Now common practise in experiments with light radioactive beams is to monitor the beam and target via scattered deuterons in an annular Si detector downstream of the target as shown in Fig. 3. At the back of HELIOS is a recoil detector, providing unambiguous gating on the $(d, p)$ events of interest, and a zero-degree telescope for determining the beam intensity.

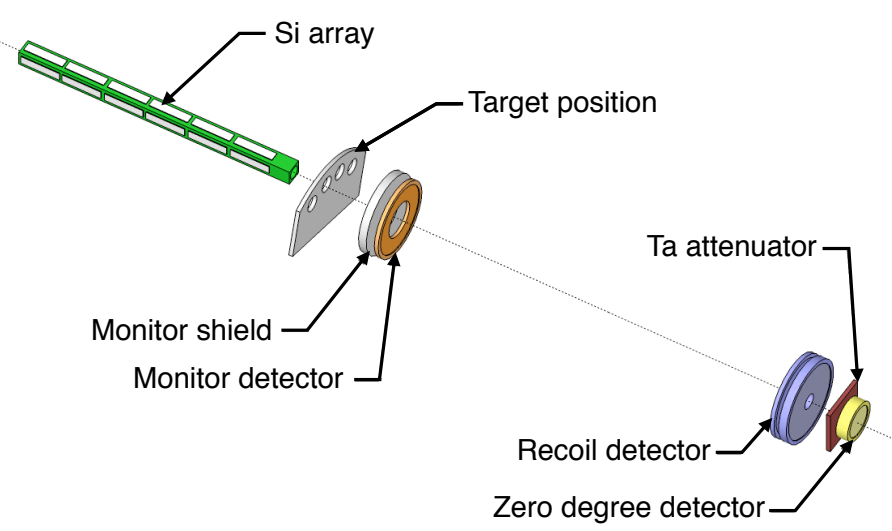

Fig. 3. Schematic of the generic experimental set up inside the solenoid for measurements with in-flight produced beams. Figure slightly modified from Ref. [22].

The $d\left({ }^{15} \mathrm{C}, p\right)$ reaction [18] sought to address the questions raised about the electromagnetic transition rates for the $2_{1}^{+} \rightarrow 0^{+}$. There were results showing anomalously small $B(E 2)[19,20]$ values suggesting 'exotic' behaviour in ${ }^{16} \mathrm{C}$, with a more recent, conflicting result consistent with a shell-model description of ${ }^{16} \mathrm{C}[21]$. The $d\left({ }^{15} \mathrm{C}, p\right)$ reaction was studied at $8.2 \mathrm{MeV} / u$ and $2.85 \mathrm{~T}$ with the beam impinging a $110-\mu \mathrm{g} / \mathrm{cm}^{2}$ thick $\mathrm{CD}_{2}$ target yielding a $Q$-value resolution of $140 \mathrm{keV}$ [18]. The measured spectroscopic factors corroborate findings from Ref. [21] that ${ }^{16} \mathrm{C}$ is a good shellmodel nucleus. 
Other studies with in-flight beams include the $d\left({ }^{19} \mathrm{O}, p\right)$ reaction which was a significant expansion on knowledge of states in ${ }^{20} \mathrm{O}$ [22] and that of the $d\left({ }^{17} \mathrm{~N}, p\right)$ reaction, which is still under analysis. These measurements have demonstrated that HELIOS can be used for detailed studies of light $s d$-shell nuclei. More recent measurements have started to exploit the proton-removing $\left(d,{ }^{3} \mathrm{He}\right)$ reaction, where the outgoing ions go forward in HELIOS. This built upon a test with a stable beam ${ }^{28} \mathrm{Si}$ [12].

\subsection{Heavier stable beams in anticipation of CARIBU}

In the near future, the CAlifornium Radioactive Isotope Breeder Upgrade (CARIBU) [23] at the ATLAS facility will allow the re-acceleration of ${ }^{252} \mathrm{Cf}$ fission fragments. In anticipation of this, we carried out the $d\left({ }^{86} \mathrm{Kr}, p\right)$ [24] and $d\left({ }^{136} \mathrm{Xe}, p\right)[25]$ reactions at $10 \mathrm{MeV} / u$. The former is close to the lightest beam that will be available from CARIBU, while the latter is close to the ${ }^{132} \mathrm{Sn}$ region, where there is major interest in transfer studies - as such this was an ideal test case for the HELIOS spectrometer. In both cases the evolution of the high- $j$ single-neutron states was the focus: at $N=51$, the evolution of the $g_{7 / 2}$ and $h_{11 / 2}$ orbitals; for $N=83$, the $h_{9 / 2}$ and $i_{13 / 2}$ orbitals. The latter had previously been studied for $56 \leq Z \leq 62$ [26], and found to behave in a manner consistent with the action of the tensor force.

Both reactions had been studied before, using gas-cell targets, and for ${ }^{136}$ Xe inverse kinematics as mentioned earlier [1] though little detailed information is known about ${ }^{137} \mathrm{Xe}$. The experiments were both carried out with a 2 -T field. Figure 4 shows the outgoing proton spectra from these
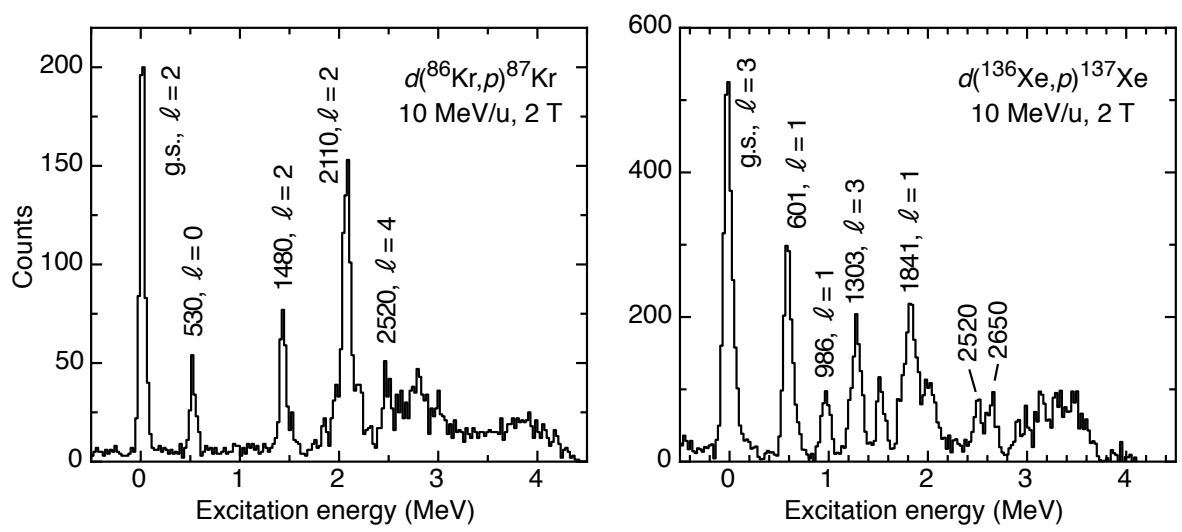

Fig. 4. Outgoing proton spectra following the $(d, p)$ reaction on ${ }^{86} \mathrm{Kr}$ (left) and ${ }^{136} \mathrm{Xe}$ (right). Spectra modified from Refs. [24] and [25], respectively. The peak energies are labelled in $\mathrm{keV}$. 
reactions. For the $(d, p)$ reaction on ${ }^{86} \mathrm{Kr}$, a $Q$-value resolution of $\sim 70 \mathrm{keV}$ was achieved with targets of thickness $70-100 \mu \mathrm{g} / \mathrm{cm}^{2}$. For ${ }^{137} \mathrm{Xe}$ the resolution was $\sim 100 \mathrm{keV}$. Several new states were observed along with the confirmation of the energy and $\ell$ values of the lowest-lying $9 / 2^{-}$and $13 / 2^{+}$ states.

Of particular interest will be $(d, p)$-reaction studies with beams in the vicinity of ${ }^{132} \mathrm{Sn}$, with a possible first measurement being with a ${ }^{134} \mathrm{Te}$ beam - one of the most intense from CARIBU. There have been several singleneutron-adding reactions using ${ }^{9} \mathrm{Be}$ and ${ }^{13} \mathrm{C}$ targets and a ${ }^{134} \mathrm{Te}$ beam carried out at Oak Ridge [27, 28]. The $(d, p)$ reaction has also been measured at $4.8 \mathrm{MeV} / u$ by Pain et al. [29] utilising the ORRUBA array at ORNL to focus on low- $\ell$ transfer and extract spectroscopic factors. At present, only limited information is available and a higher energy $(d, p)$ measurement is desired. Knowledge of the energies, spins and spectroscopic factors of states in ${ }^{135} \mathrm{Te}$ are important as the evolution of single-particle energies across the $N=83$ isotones approaches ${ }^{133} \mathrm{Sn}$.

\section{Summary and outlook}

HELIOS represents a solution to the challenge of studying transfer reactions in inverse kinematics, complementing other approaches such as $\mathrm{Si}$ barrel arrays in conjunction with gamma-ray detectors. At ANL, the device has demonstrated excellent $Q$-value resolution in studies with light neutronrich beams and promising results with medium-mass stable beams comparable to those expected from the CARIBU upgrade at ATLAS. Studies of neutron-rich nuclei in the ${ }^{132} \mathrm{Sn}$ region are eagerly anticipated.

Many new improvements are expected over the coming year, with the development of a gas-cell target for astrophysical studies and a fast ionisation chamber for recoil detection. The Si array is also due for an update, increasing the acceptance by a factor of about two.

In Europe, there is interest in the development of a solenoidal spectrometer in anticipation of HIE-ISOLDE and SPIRAL2. Possible use of such a device coupled with the cooled beams of a storage ring have been discussed as part of the TSR project [30].

This work was supported by the U.S. Department of Energy, Office of Nuclear Physics, under Contract No. DE-AC02-06CH11357 (ANL) and Grant No. DE-FG02-04ER41320 (WMU), NSF Grant No. PHY-08022648 (JINA), and the UK Science and Technology Facilities Council. 


\section{REFERENCES}

[1] G. Kraus et al., Z. Phys. A340, 339 (1991).

[2] K.E. Rehm et al., Phys. Rev. Lett. 80, 676 (1998).

[3] K.L. Jones et al., Nature 465, 454 (2010).

[4] M. Labiche et al., Nucl. Instrum. Methods Phys. Res. A614, 439 (2010).

[5] C.Aa. Diget et al., J. Instrum. 6, P02005 (2011).

[6] S.D. Pain et al., Nucl. Instrum. Methods Phys. Res. B261, 1122 (2007).

[7] V. Bildstein et al., Prog. Part. Nucl. Phys. 59, 386 (2007).

[8] Y. Blumenfeld et al., Nucl. Instrum. Methods Phys. Res. A421, 471 (1999).

[9] E. Pollacco et al., Eur. Phys. J.A 25, 287 (2005).

[10] A.H. Wuosmaa et al., Nucl. Instrum. Methods Phys. Res. A580, 1290 (2007).

[11] D.W. Bardayan et al., Phys. Rev. C78, 052801(R) (2008).

[12] B.P. Kay et al., J. Phys.: Conf. Ser. 381, 012095 (2012).

[13] A. Micalowicz, Kinematics of Nuclear Reactions, London: Iliffe, 1967.

[14] J.C. Lighthall et al., Nucl. Instrum. Methods Phys. Res. A622, 97 (2010).

[15] B. Harss et al., Rev. Sci. Instrum. 71, 380 (2000).

[16] B.B. Back et al., Phys. Rev. Lett. 104, 132501 (2010).

[17] H.Y. Lee et al., Phys. Rev. C81, 015802 (2010).

[18] A.H. Wuosmaa et al., Phys. Rev. Lett. 105, 132501 (2010).

[19] Z. Elekes et al., Phys. Lett. 586, 34 (2004).

[20] N. Imai et al., Phys. Rev. Lett. 92, 062501 (2004).

[21] M. Wiedeking et al., Phys. Rev. Lett. 100, 152501 (2008).

[22] C.R. Hoffman et al., Phys. Rev. C85, 054318 (2012).

[23] G. Savard et al., Nucl. Instrum. Methods Phys. Res. B266, 4086 (2008).

[24] D.K. Sharp et al., Phys. Rev. C87, 014312 (2013).

[25] B.P. Kay et al., Phys. Rev. C84, 024325 (2011).

[26] B.P. Kay et al., Phys. Lett. B658, 216 (2008).

[27] D.C. Radford et al., Eur. Phys. J. A25, 383 (2005).

[28] J.M. Allmond et al., Phys. Rev. C86, 031307(R) (2012).

[29] J.A. Cizewski et al., J. Phys.: Conf. Ser. 239, 012007 (2010).

[30] M. Grieser et al., Eur. Phys. J. Special Topics 207, 1 (2012). 